\title{
Analytic Singular Support Properties for Integral Operators in Hyperfunctions
}

\author{
by \\ Otto LIEss and Yasunori OKADA
}

\begin{abstract}
In this paper, we continue our study of integral operators in [12] and give a result on the analytic singular support of the kernel hyperfunction similar to the main result in [12]. A new feature with respect to [12] is that we use classes of ultradifferentiable functions associated with a weight function and a topological description of related spaces of quasianalytic functionals.
\end{abstract}

2010 Mathematics Subject Classification: Primary 46F12; Secondary 46F15. Keywords: integral transforms, hyperfunctions.

\section{$\S 1$. Introduction}

In this paper, we continue the study of integral operators begun in [12] and give a result on the analytic singular support of the kernel hyperfunction. The main theorem in [12] concerned support properties of integral operators, whereas our present result will concern similar singular support properties.

We recall here some notation and explain the setting. Consider open sets $U \subset \mathbb{R}^{m}, V \subset \mathbb{R}^{n}$, and a hyperfunction $\mathcal{K}$ defined on $V \times U$ which satisfies the condition

$$
\left\{(x, y, 0, \eta) \in V \times U \times \mathbb{R}^{n} \times \mathbb{R}^{m} ; \eta \neq 0\right\} \cap \mathrm{WF}_{A} \mathcal{K}=\emptyset,
$$

where $\mathrm{WF}_{A} \mathcal{K}$ denotes the analytic wave front set of $\mathcal{K}$. We can then associate with $\mathcal{K}$ a linear operator $T: \mathcal{A}^{\prime}(U) \rightarrow \mathcal{B}(V)$ by

$$
(T u)(x)=\int_{U} \mathcal{K}(x, y) u(y) d y \quad \text { for } u \in \mathcal{A}^{\prime}(U) .
$$

Communicated by T. Mochizuki. Received March 2, 2014. Revised July 15, 2015.

O. Liess: Department of Mathematics, University of Bologna, Bologna, 40127, Italy; e-mail: ottoedwin.liess@unibo.it

Y. Okada: Department of Mathematics and Informatics, Graduate School of Science, Chiba University, Inage-ku, Chiba 263-8522, Japan;

e-mail: okada@math.s.chiba-u.ac.jp

(C) 2015 Research Institute for Mathematical Sciences, Kyoto University. All rights reserved. 
Here $\mathcal{A}^{\prime}(U)$ denotes the space of real-analytic functionals on $U, \mathcal{B}(V)$ the space of hyperfunctions on $V$, and the meaning of the integral in (1.2) is the one given by microlocal analysis to such expressions (see Sato-Kawai-Kashiwara [16]). Note that we shall identify $\mathcal{A}^{\prime}(U)$ with the space $\mathcal{B}_{c}(U)$ of hyperfunctions on $U$ with compact support. In this setting, $T$ is said to be the integral operator associated with $\mathcal{K}$, and $\mathcal{K}$ is said to be the kernel of $T$.

Our main result is the following

Theorem 1.1. Let $T: \mathcal{A}^{\prime}(U) \rightarrow \mathcal{B}(V)$ be the integral operator associated with some kernel $\mathcal{K} \in \mathcal{B}(V \times U)$ satisfying (1.1). Assume that $\operatorname{singsupp}_{A} T u \subset V$ is compact for every $u \in \mathcal{A}^{\prime}(U)$. Then the projection $(V \times U) \cap \operatorname{singsupp}_{A} \mathcal{K} \rightarrow U$ is proper.

Here and later on, $\operatorname{singsupp}_{A} u$ denotes the analytic singular support of $u$. We mention that [12, Theorem 1.1] had a statement which was quite similar to that of Theorem 1.1, in that it stated that if for an operator as in (1.2), supp Tu is compact for every $u \in \mathcal{A}^{\prime}(U)$, then the projection $(V \times U) \cap \operatorname{supp} \mathcal{K} \rightarrow U$ must be proper.

Classical examples of operators which are as in Theorem 1.1 include the operators associated with parametrices for analytic pseudodifferential operators and analytic Fourier integral operators.

The argument in [12] was based on a preliminary decomposition of the kernel $\mathcal{K}$ in terms of $C^{\infty}$ functions. By studying non-analyticity of differentiable functions in a quantitative way we could avoid cohomological characterizations of the supports of the hyperfunctions in the image of $T$. A similar technique is again needed in this paper, but here we shall use related decompositions of $\mathcal{K}$ with respect to an adapted class of possibly quasianalytic ultradifferentiable functions and we must then know that any real-analytic functional will be in one of the spaces of quasianalytic functionals associated with some class. The difference between the approaches in [12] and the present paper comes from the fact that to characterize the support of some hyperfunction is easier than to characterize its singular support. See Remark 6.5 for more details on what we mean by this statement. As for the classes of ultradifferentiable functions and quasianalytic functionals, we will use those introduced and studied by Braun-Meise-Taylor [5] and Heinrich-Meise [6].

The plan of the paper is as follows. In Section 2, we recall the notion of ultradifferentiable classes with respect to weight functions and quasianalytic functionals, from [5] and [6]. These classes will be called BMT-classes for short. We also cite a topological description of the spaces of quasianalytic functionals via the Fourier-Laplace transform, and an existence result for weight functions which may be large at infinity (see Theorem 2.7, Lemma 2.8 and the citations there). 
In Section 3, we study twisted Radon transforms applied to ultradifferentiable functions. There we shall see that an important feature of BMT-classes is that they are stable under twisted Radon decompositions (Lemma 3.2). This fact will then lead to boundary value representations for ultradifferentiable kernels (Theorem 3.4). When our kernel functions $\mathcal{K}$ in (1.2) are ultradifferentiable and when they operate on quasianalytic functionals, then the integrals there can also be given a meaning by duality. We have thus two ways of defining our integral operators, an initial one in terms of integration of hyperfunctions as in [16] and a second one by duality. Based on the result in Section 3, we establish in Section 4 that the two interpretations of the integral (1.2) are compatible (Corollary 4.1).

In Section 5, after showing in Lemma 5.2 that the inverse Fourier transforms of functions satisfying suitable decay estimates are ultradifferentiable, we prove a division theorem for a hyperfunction kernel. In it, a given kernel is written, up to a real-analytic remainder, in the form $P\left(D_{x}\right) \mathcal{K}^{\prime}$, where $P\left(D_{x}\right)$ is a possibly infinite order elliptic partial differential operator in the $x$ variable and $\mathcal{K}^{\prime}$ is an ultradifferentiable quotient (Theorem 5.1). Due to this, we can then give, in the final Section 6, the proof of our main result, Theorem 1.1, also using the fact that the space of real-analytic functionals is exhausted by the classes of quasianalytic functionals which appear in BMT theory.

We conclude the introduction by recalling [12, Theorems 1.2 and 1.3].

Theorem 1.2. Let $\mathcal{K} \in \mathcal{B}(V \times U)$ be a hyperfunction satisfying (1.1) and let $T$ be the associated operator defined in (1.2). If $T u=0$ for every $u \in \mathcal{A}^{\prime}(U)$, then $\mathcal{K}$ must vanish on $V \times U$.

Theorem 1.3. Let $\mathcal{K} \in \mathcal{B}(V \times U)$ be a hyperfunction satisfying (1.1). Assume that the operator $T: \mathcal{A}^{\prime}(U) \rightarrow \mathcal{B}(V)$ defined in (1.2) actually maps $\mathcal{A}^{\prime}(U)$ into $\mathcal{A}(V)$. Then $\mathcal{K}$ is real-analytic on $V \times U$.

The two theorems are essentially consequences of results of Kaneko [9], BastinLaubin [2, 3] and Kataoka-Oshima (see [8, Theorem 4.4.7'] for the latter). For the reason, see [12, Remark 1.7 and $\S 2]$.

\section{$\S 2$. Ultradifferentiability with respect to a weight function}

We now recall part of the terminology and of the results on the notion of ultradifferentiability with respect to weight functions, and on quasianalytic functionals associated with weight functions, as introduced by Braun-Meise-Taylor [5] and Heinrich-Meise [6]. For the relation between this and the notions of ultradiffer- 
entiability given by sequences, we refer to Bonet-Meise-Melikhov [4]. Also refer to [13] for the relation to so-called inhomogeneous Gevrey classes of [15].

First we give the notion of a Young conjugate (or Legendre transform) for increasing functions defined on $[0, \infty[$.

Definition 2.1 ([4, Definition 2.3]). Let $\varphi:[0, \infty[\rightarrow[0, \infty[$ be an increasing function satisfying $\varphi(0)=0$ and $x=o(\varphi(x))$ as $x \rightarrow \infty$. Then the Young conjugate $\varphi^{*}$ of $\varphi$ is defined as

$$
\varphi^{*}:\left[0, \infty\left[\rightarrow \left[0, \infty\left[, \quad \varphi^{*}(y):=\sup _{x \geq 0}(x y-\varphi(x)) .\right.\right.\right.\right.
$$

We also use the notation

$$
\varphi_{[\lambda]}^{*}(y):=\lambda \varphi^{*}(y / \lambda)
$$

which is the Young conjugate of $\lambda \varphi(\cdot)$, for $\lambda>0$. Therefore, the family $\{\lambda \varphi\}_{\lambda>0}$ corresponds to the family $\left\{\varphi_{[\lambda]}^{*}\right\}_{\lambda>0}$.

Definition 2.2 ([4, Definition 2.1(a)]). Let $\omega:[0, \infty[\rightarrow[0, \infty[$ be a continuous increasing function which satisfies $\left.\omega\right|_{[0,1]} \equiv 0$. It is called a weight function if it has the following properties:

$(\alpha) \omega(2 t)=O(\omega(t))$ as $t \rightarrow \infty$,

( $\beta) \log (1+t)=o(\omega(t))$ as $t \rightarrow \infty$,

$(\gamma) \omega(t)=O(t)$ as $t \rightarrow \infty$,

$(\delta) \varphi:\left[0, \infty\left[\rightarrow\left[0, \infty\left[\right.\right.\right.\right.$ defined by $\varphi(x):=\omega\left(e^{x}\right)$ is convex.

A weight function $\omega(t)$ is called quasianalytic if $\int_{0}^{\infty} \frac{\omega(t)}{1+t^{2}} d t=\infty$. While we shall mainly work with quasianalytic cases, we shall not use properties specific to quasianalyticity.

For a weight function $\omega$, we always consider the function $\varphi$ defined in Definition $2.2(\delta)$ and its Young conjugate $\varphi^{*}$. Note that for a weight function $\omega$, the $\varphi$ given in Definition 2.2( $\delta$ ) always satisfies the hypothesis of Definition 2.1. In fact, $x=o(\varphi(x))$ as $x \rightarrow \infty$ in view of Definition $2.2(\beta)$.

Example 2.3. The function $\omega_{\mathrm{A}}(t):=\max \{0, t-1\}$ is a weight function corresponding to $\varphi_{\mathrm{A}}(x)=e^{x}-1$ with Young conjugate $\varphi_{\mathrm{A}}^{*}(y)=y \log y-y+1$.

Consider two weight functions $\omega_{1}$ and $\omega_{2}$, and let $\varphi_{j,[\lambda]}^{*}$ for $\lambda>0$ and $j=$ 1,2 denote the functions given by $(2.1)$ and $(2.2)$ for $\varphi_{j}(x):=\omega_{j}\left(e^{x}\right)(j=1,2)$ respectively. Then

$$
\forall t \geq 0, \omega_{1}(t) \leq \omega_{2}(t) \Rightarrow \forall y \geq 0, \varphi_{1,[\lambda]}^{*}(y) \geq \varphi_{2,[\lambda]}^{*}(y)
$$


Proposition 2.4. Let $\omega$ be a weight function. Define $\varphi, \varphi^{*}$ and $\varphi_{[\lambda]}^{*}$ by Definition 2.2( $\delta),(2.1)$ and $(2.2)$. Then:

(a) $\varphi^{*}$ is a convex increasing function mapping $\left[0, \infty\left[\right.\right.$ into $\left[0, \infty\left[\right.\right.$, satisfying $\varphi^{*}(0)$ $=0$ and $\lim _{y \rightarrow \infty} \varphi^{*}(y) / y=\infty$.

(b) $\left(\varphi^{*}\right)^{*}=\varphi$; more generally, $\left(\varphi_{[\lambda]}^{*}\right)^{*}=\lambda \varphi$.

(c) $\varphi_{[\lambda]}^{*}(y)$ is decreasing in $\lambda$, that is, $\varphi_{[\lambda]}^{*}(y) \geq \varphi_{[\mu]}^{*}(y)$ for $0<\lambda \leq \mu, y \geq 0$.

(d) $\varphi_{[\lambda]}^{*}(p)+\varphi_{[\lambda]}^{*}(q) \leq \varphi_{[\lambda]}^{*}(p+q)$ for any $\lambda>0$ and $p, q \geq 0$.

(e) $\varphi_{[\lambda+\mu]}^{*}(p+q) \leq \varphi_{[\lambda]}^{*}(p)+\varphi_{[\mu]}^{*}(q)$ for any $\lambda, \mu>0$ and $p, q \geq 0$.

(f) $\lambda \varphi(x)-x \leq \sup _{p \in \mathbb{N}_{0}}\left(x p-\varphi_{[\lambda]}^{*}(p)\right) \leq \lambda \varphi(x)$ for any $\lambda>0$ and $x \geq 0$.

(g) For any $k, l_{1}, l_{2} \geq 0$ and $\lambda>0$, there exist $\mu>0$ and $C \geq 0$ such that

$$
\varphi_{[\lambda]}^{*}\left(y+l_{1}\right)+k y \leq \varphi_{[\mu]}^{*}\left(y+l_{2}\right)+C, \quad y \geq 0 .
$$

On the other hand, for any $k, l_{1}, l_{2} \geq 0$ and $\lambda>0$, there exist $\mu>0$ and $C \geq 0$ such that

$$
\varphi_{[\lambda]}^{*}\left(y+l_{1}\right)-k y \geq \varphi_{[\mu]}^{*}\left(y+l_{2}\right)-C, \quad y \geq 0 .
$$

(See [6, Lemma 2.6].)

(h) If $\omega$ is sublinear, i.e., $\omega(t)=o(t)$ as $t \rightarrow \infty$, then

$$
\varphi_{[\lambda]}^{*}(y) / y-\log y \rightarrow \infty \quad \text { as } y \rightarrow \infty,
$$

for any $\lambda>0$. In particular, $\inf _{y>0}\left(\varphi_{[\lambda]}^{*}(y)-y \log y-a y\right)>-\infty$ for any sublinear $\omega, \lambda>0$ and $a \in \mathbb{R}$.

Here in (f) and later on, $\mathbb{N}_{0}$ denotes $\{0,1,2, \ldots\}$, while $\mathbb{N}=\{1,2, \ldots\}$.

Proof. (a) and (b). That $\varphi^{*}$ is increasing and $\varphi^{*}(0)=0$ follows directly from the definition. The convexity of $\varphi^{*}$ follows from the fact that $\varphi^{*}$ is the supremum of the convex functions $y \mapsto x y-\varphi(x)$. By definition, and from the convexity of $\varphi$,

$$
\varphi^{* *}(x)=\sup _{y \geq 0}\left(x y-\varphi^{*}(y)\right)=\sup _{y \geq 0} \inf _{p \geq 0}((x-p) y+\varphi(p))=\varphi(x) .
$$

This also implies $\varphi^{*}(y) / y \geq x-\varphi(x) / y$ for any $x, y \geq 0$, therefore we have $\lim _{y \rightarrow \infty} \varphi^{*}(y) / y=\infty$, or $y=o\left(\varphi^{*}(y)\right)$ as $y \rightarrow \infty$.

(c), (d) and (e) follow from the convexity of $\varphi^{*}$ and $\varphi^{*}(0)=0$. In fact, they follow from the intermediate inequalities

$$
\begin{gathered}
\varphi^{*}(y / \mu) \leq(1-\lambda / \mu) \varphi^{*}(0)+(\lambda / \mu) \varphi^{*}(y / \lambda) \quad \text { for } 0<\lambda \leq \mu, \\
\varphi_{[\lambda]}^{*}(p) \leq \frac{q \varphi_{[\lambda]}^{*}(0)+p \varphi_{[\lambda]}^{*}(p+q)}{p+q}, \quad \varphi_{[\lambda]}^{*}(q) \leq \frac{p \varphi_{[\lambda]}^{*}(0)+q \varphi_{[\lambda]}^{*}(p+q)}{q+p},
\end{gathered}
$$




$$
\varphi^{*}\left(\frac{\lambda(p / \lambda)+\mu(q / \mu)}{\mu+\lambda}\right) \leq \frac{\lambda \varphi^{*}(p / \lambda)+\mu \varphi^{*}(q / \mu)}{\mu+\lambda},
$$

which are consequences of the convexity of $\varphi^{*}$ and $\varphi_{[\lambda]}^{*}$.

(f) for the general $\lambda>0$ follows from the special case $\lambda=1$. The right inequality follows trivially from (b) so let us prove the left one. Denoting by $\lfloor y\rfloor$ the integer part of $y$, we obtain $x y-\varphi^{*}(y) \leq x(\lfloor y\rfloor+1)-\varphi^{*}(\lfloor y\rfloor)$ since $\lfloor y\rfloor \leq y<\lfloor y\rfloor+1$ and $\varphi^{*}$ is increasing. By taking the supremum in $y \geq 0$ and using (b), we have

$$
\varphi(x) \leq \sup _{p \in \mathbb{N}_{0}}\left(x(p+1)-\varphi^{*}(p)\right)=\sup _{p \in \mathbb{N}_{0}}\left(x p-\varphi^{*}(p)\right)+x .
$$

(g) was proved in [6, Lemma 2.6], but let us prove it also here for the convenience of the reader. Note that from (e) we have, for any $\lambda>\mu>0$ and any $l_{1}, l_{2} \geq 0$,

$$
\sup _{y \geq 0}\left(\varphi_{[\lambda]}^{*}\left(y+l_{1}\right)-\varphi_{[\mu]}^{*}\left(y+l_{2}\right)\right)<\infty .
$$

In fact, the left hand side is less than $\varphi_{[\lambda-\mu]}^{*}\left(l_{1}-l_{2}\right)$ when $l_{1} \geq l_{2}$, and zero otherwise. Note also that from Definition 2.2( $\alpha)$, there exists a constant $A>1 \mathrm{such}$ that $\varphi(x+1) \leq A(\varphi(x)+1)$ for any $x \geq 0$, which implies $\varphi(x+k) \leq A^{k}(\varphi(x)+k)$ for $k \in \mathbb{N}_{0}$ and $x \geq 0$. Thus

$$
\begin{aligned}
\varphi_{[\lambda]}^{*}\left(y+l_{1}\right)+k y & =\sup _{x \geq 0}\left(x\left(y+l_{1}\right)-\lambda \varphi(x)\right)+k y \\
& =\sup _{x \geq 0}\left((x+k)\left(y+l_{1}\right)-k l_{1}-\lambda(\varphi(x)+k)+k \lambda\right) \\
& \leq k\left(\lambda-l_{1}\right)+\sup _{x \geq 0}\left((x+k)\left(y+l_{1}\right)-\lambda A^{-k} \varphi(x+k)\right) \\
& \leq k\left(\lambda-l_{1}\right)+\varphi_{\left[\lambda / A^{k}\right]}^{*}\left(y+l_{1}\right) .
\end{aligned}
$$

If we choose $\mu$ with $0<\mu<\lambda / A^{k}$, then (2.3) follows from (2.5). Similarly,

$$
\begin{aligned}
\varphi_{[\lambda]}^{*}\left(y+l_{1}\right)-k y & =\sup _{x \geq 0}\left(x\left(y+l_{1}\right)-\lambda \varphi(x)\right)-k y \\
& \geq \sup _{x \geq k}\left((x-k)\left(y+l_{1}\right)+k l_{1}-\lambda \varphi(x)\right) \\
& \geq k l_{1}+\sup _{x \geq k}\left((x-k)\left(y+l_{1}\right)-\lambda A^{k} \varphi(x-k)-\lambda k A^{k}\right) \\
& =k\left(l_{1}-k A^{k}\right)+\varphi_{\left[\lambda A^{k}\right]}^{*}\left(y+l_{1}\right) .
\end{aligned}
$$

If we choose $\mu$ with $\mu>\lambda A^{k}$, then (2.4) follows from (2.5).

The second statement of (h) follows from the first one, which we will prove. The inequality $\varphi(x) \leq A e^{x}+B$ for $x \geq 0$ and positive constants $A$ and $B$ implies

$$
\varphi^{*}(y) \geq \sup _{x \geq 0}\left(x y-A e^{x}-B\right) \geq y \log (y / A)-y-B \quad \text { for } y \geq A,
$$


and therefore $\liminf \inf _{y \rightarrow \infty}\left(\varphi^{*}(y) / y-\log y\right) \geq-1-\log A$. Since $\varphi(x)=o\left(e^{x}\right)$ from the sublinearity of $\omega$, we can take an arbitrary $A>0$, which concludes the proof for $\lambda=1$. In view of

$$
\varphi_{[\lambda]}^{*}(y) / y-\log y=\varphi^{*}(y / \lambda) /(y / \lambda)-\log (y / \lambda)-\log \lambda,
$$

the general case follows from the case $\lambda=1$.

Definition 2.5. Let $\omega$ be a weight function and $V \subset \mathbb{R}^{n}$ an open set. Then we define, for $\lambda>0$ and $K \Subset V$, a quasi-norm $p_{\omega, \lambda, K}$ on $C^{\infty}(V)$ by

$$
p_{\omega, \lambda, K}(f):=\sup _{x \in K, \alpha \in \mathbb{N}_{0}^{n}}\left|\partial^{\alpha} f(x)\right| \exp \left(-\varphi_{[\lambda]}^{*}(|\alpha|)\right),
$$

and also define the spaces $\mathcal{E}_{(\omega)}(V)$ and $\mathcal{E}_{\{\omega\}}(V)$ of $\omega$-ultradifferentiable functions of Beurling type, respectively of Roumieu type, on $V$ by

$$
\begin{aligned}
& \mathcal{E}_{(\omega)}(V):=\left\{f \in C^{\infty}(V) ; \forall K \Subset V, \forall m \in \mathbb{N}, p_{\omega, m, K}(f)<\infty\right\}, \\
& \mathcal{E}_{\{\omega\}}(V):=\left\{f \in C^{\infty}(V) ; \forall K \Subset V, \exists m \in \mathbb{N}, p_{\omega, 1 / m, K}(f)<\infty\right\} .
\end{aligned}
$$

These spaces are endowed with the natural locally convex topologies given by

$$
\begin{aligned}
& \mathcal{E}_{(\omega)}(V)=\lim _{K \Subset V} \lim _{m \in \mathbb{N}}\left\{f \in C^{\infty}(V) ; p_{\omega, m, K}(f)<\infty\right\}, \\
& \mathcal{E}_{\{\omega\}}(V)=\lim _{K \Subset V} \underset{m \in \mathbb{N}}{\lim _{m \in \mathbb{N}}}\left\{f \in C^{\infty}(V) ; p_{\omega, 1 / m, K}(f)<\infty\right\},
\end{aligned}
$$

where $\lim _{\longrightarrow}$ and $\varliminf_{\text {lim }}$ denote the inductive and projective limits respectively. We denote by $\overrightarrow{\mathcal{E}}_{(\omega)}^{\prime}(V)$ and $\mathcal{E}_{\{\omega\}}^{\prime}(V)$ the respective strong dual spaces, and their elements are called $(\omega)$-ultradistributions (respectively $\{\omega\}$-ultradistributions) with compact support in $V$. If $\omega$ is quasianalytic, they are also called quasianalytic functionals of $(\omega)$-type (respectively of $\{\omega\}$-type) on $V$.

By definition, we have the following continuous inclusion maps for weight functions $\omega$ and $\tilde{\omega}$ :

$$
\begin{aligned}
& \mathcal{E}_{(\omega)}(V) \subset \mathcal{E}_{\{\omega\}}(V), \\
\mathcal{E}_{(\tilde{\omega})}(V) \subset \mathcal{E}_{(\omega)}(V), \quad & \mathcal{E}_{\{\tilde{\omega}\}}(V) \subset \mathcal{E}_{\{\omega\}}(V) \quad \text { if } \omega=O(\tilde{\omega}) \text { as } t \rightarrow \infty, \\
& \mathcal{E}_{\{\tilde{\omega}\}}(V) \subset \mathcal{E}_{(\omega)}(V) \quad \text { if } \omega=o(\tilde{\omega}) \text { as } t \rightarrow \infty .
\end{aligned}
$$

For example, if we take $\omega_{\mathrm{A}}$ given in Example 2.3, then $\mathcal{E}_{\left\{\omega_{\mathrm{A}}\right\}}(V) \simeq \mathcal{A}(V)$ as locally convex spaces, and therefore $\mathcal{A}(V)$ is continuously included in $\mathcal{E}_{(\omega)}(V)$ and in $\mathcal{E}_{\{\omega\}}(V)$ for any sublinear weight function $\omega$. If moreover $V$ is connected, then $\mathcal{E}_{\left(\omega_{\mathrm{A}}\right)}(V) \simeq \mathcal{O}\left(\mathbb{C}^{n}\right)$. From now on, we restrict ourselves to considering $\mathcal{E}_{(\omega)}(V)$ and $\mathcal{E}_{(\omega)}^{\prime}(V)$ only when $\omega$ is sublinear. 
Note that $p_{\omega, \lambda, K}(f)$ increases in $\lambda$ (see Proposition 2.4(c)). Therefore we may take any unbounded subset in $\mathbb{N}$ as a cofinal set in the projective and inductive limits in $m$ in (2.6) and in (2.7) respectively. Also note that the inequality in Proposition 2.4(e) with $\lambda=\mu$ shows that

$$
p_{\omega, \lambda, K}\left(\partial^{\beta} f\right) \leq \exp \left(\varphi_{[\lambda]}^{*}(|\beta|)\right) \cdot p_{\omega, 2 \lambda, K}(f),
$$

which proves the stability of $\mathcal{E}_{(\omega)}(V)$ and $\mathcal{E}_{\{\omega\}}(V)$ under derivation by $\partial^{\beta}$.

Let $*$ denote $(\omega)$ or $\{\omega\}$. The spaces $\mathcal{E}_{*}^{\prime}(V)$ are canonically embedded in $\mathcal{A}^{\prime}(V)$ if $*=(\omega)$ and $\omega(t)=o(t)$, or if $*=\{\omega\}$, since $\mathcal{A}(V)$ is dense in $\mathcal{E}_{*}(V)$ (see $[6$, Proposition 3.2]).

Definition 2.6 ([6, Definition 3.4]). Let $\omega$ be a weight function and $V \subset \mathbb{R}^{n}$ an open convex set. For a compact set $K \Subset V$, we denote the supporting function of $K$ by

$$
H_{K}(\xi):=\sup _{x \in K}\langle x, \xi\rangle,
$$

and define Banach spaces $A(K, \lambda)$ for $\lambda>0$ by

$$
A(K, \lambda):=\left\{f \in \mathcal{O}\left(\mathbb{C}^{n}\right) ;\|f\|_{K, \lambda}:=\sup _{\zeta \in \mathbb{C}^{n}}|f(\zeta)| \exp \left(-H_{K}(\operatorname{Im} \zeta)-\lambda \omega(|\zeta|)\right)<\infty\right\} .
$$

We also define the locally convex spaces

$$
A_{(\omega)}\left(\mathbb{C}^{n}, V\right):=\underset{K \Subset V}{\lim _{k \in \mathbb{N}}} \lim _{k} A(K, k), \quad A_{\{\omega\}}\left(\mathbb{C}^{n}, V\right):=\underset{K \Subset V}{\lim _{k \in \mathbb{N}}} \lim _{k} A(K, 1 / k) .
$$

When $V$ is convex and $\omega$ is sublinear, the Fourier-Laplace transform

$$
\mathcal{F}: \mathcal{A}^{\prime}(V) \ni u \mapsto \hat{u} \in \mathcal{O}\left(\mathbb{C}^{n}\right), \quad \hat{u}(\zeta):=\left\langle u, e^{-i\langle\cdot, \zeta\rangle}\right\rangle,
$$

induces the following topological isomorphisms:

Theorem 2.7 ([6, Theorems 3.6 and 3.7]). Let $\omega$ be a sublinear weight function and $V \subset \mathbb{R}^{n}$ an open convex set. Then the linear maps

$$
\mathcal{F}: \mathcal{E}_{(\omega)}^{\prime}(V) \rightarrow A_{(\omega)}\left(\mathbb{C}^{n}, V\right), \quad \mathcal{F}: \mathcal{E}_{\{\omega\}}^{\prime}(V) \rightarrow A_{\{\omega\}}\left(\mathbb{C}^{n}, V\right)
$$

are isomorphisms between the corresponding locally convex spaces.

The following result on existence of weight functions is a special case of $[5$, Lemma 1.7 and Remark 1.8(1)].

Lemma 2.8. For any sublinear function $\ell(t)$, there exists a sublinear weight function $\omega(t)$ such that $\ell(t)=o(\omega(t))$ as $t \rightarrow \infty$.

These results imply that every real-analytic functional belongs to some class of quasianalytic functionals associated with some sublinear weight function. 


\section{$\S 3$. Twisted Radon transforms of ultradifferentiable functions}

In this section, we will study properties of a given ultradifferentiable integral kernel using a twisted Radon transform. The main result in this section is Theorem 3.4.

Twisted Radon transforms are integral operators associated with twisted Radon kernels. Here we recall such a twisted Radon kernel in one of the forms in which such kernels appear in the literature. For notational simplicity we will recall the kernel in $n$ variables, although when we work with it we will apply it in $\mathbb{R}^{n+m}$. We use in fact a Radon kernel of Bony type:

$$
W(z, \xi)=\frac{(n-1) !}{(-2 \pi i)^{n}} \cdot \frac{1+i\langle z, \xi\rangle}{\Phi(z, \xi)^{n}}, \quad \Phi(z, \xi)=\langle z, \xi\rangle+i\langle z, z\rangle,
$$

regarded as a function defined on the set

$$
\left\{(z, \xi) \in \mathbb{C}^{n} \times S^{n-1} ; \operatorname{Im} \Phi(z, \xi)>0\right\}=\left\{|\operatorname{Im} z-\xi / 2|^{2}<1 / 4+|\operatorname{Re} z|^{2}\right\},
$$

and its integral with respect to $\xi \in S^{n-1} \cap \Delta$, with $\Delta$ a proper cone in $\mathbb{R}^{n}$ :

$$
W(z, \Delta):=\int_{S^{n-1} \cap \Delta} W(z, \xi) d \sigma(\xi)
$$

where $\sigma(\xi)$ denotes the standard surface element of $S^{n-1}$.

We recall some known results concerning $W(z, \xi)$. The following statements are valid not only for kernels of Bony type, but also for other twisted Radon kernels.

For a hyperfunction $u \in \mathcal{B}\left(\mathbb{R}^{n}\right)$ with compact support, and for a decomposition $\dot{\mathbb{R}}^{n}=\bigcup_{j=1}^{J} \Delta_{j}$ of $\dot{\mathbb{R}}^{n}=\mathbb{R}^{n} \backslash\{0\}$ into closed proper cones $\Delta_{j} \subset \dot{\mathbb{R}}^{n}$ with disjoint interiors, the integrals

$$
F_{j}(z):=\int_{\mathbb{R}^{n}} W\left(z-x, \Delta_{j}\right) u(x) d x
$$

for $j=1, \ldots, J$ define holomorphic functions on infinitesimal wedges of the form $\mathbb{R}^{n}+i \Delta_{j}^{\perp} 0$, and they give a family of defining functions of $u$, that is,

$$
u(x)=\sum_{j=1}^{J} F_{j}\left(x+i \Delta_{j}^{\perp} 0\right) .
$$

Here and later on we use the following notation: for a cone $\Delta \subset \mathbb{R}^{n}, \Delta^{\perp}$ denotes the dual cone $\operatorname{Int}\left\{s \in \mathbb{R}^{n} ; \forall \xi \in \Delta,\langle s, \xi\rangle>0\right\}$ of $\Delta$; for cones $G^{\prime} \subset G$ in $\mathbb{R}^{n}, G^{\prime}$ is said to be a strict subcone of $G$ if the closure of $G^{\prime} \cap S^{n-1}$ is included in the interior of $G$; and for an open set $V \subset \mathbb{R}^{n}$ and an open cone $G \subset \mathbb{R}^{n}$, an open set $U \subset \mathbb{C}^{n}$ is called an infinitesimal wedge of the form $V+i G 0$ if $U \subset V+i G$ and if for any 
$K \Subset V$ and any strict subcone $G^{\prime}$ of $G$, there exists $d>0$ with $U \supset K+i G^{\prime}[d]$, where $G^{\prime}[d]:=\left\{s \in G^{\prime} ;|s|<d\right\}$.

The great merit of twisted Radon integrals is that if we choose $\Delta_{j}$ suitably, then microanalyticity (microlocal regularity) of $u$ can be read off from the properties of the $F_{j}$ in a rather explicit form:

Theorem 3.1. Let $u \in \mathcal{B}\left(\mathbb{R}^{n}\right)$ be a hyperfunction with compact support. Assume that $\left(V_{0} \times \Delta_{j}\right) \cap \mathrm{WF}_{A} u=\emptyset$ for some open set $V_{0} \subset \mathbb{R}^{n}$. Then $F_{j}(z)$ given by (3.1) extends holomorphically to a neighborhood of $V_{0}$.

This theorem can be proved starting from the definition of integration of hyperfunctions and the definition of the analytic wave front set. For the theory of the twisted Radon transform, we refer to Kataoka [10], Kaneko [8] and AokiKataoka-Yamazaki [1].

It is also known that the differentiability and Gevrey regularity of $u$ are reflected in the corresponding properties of the $F_{j}$. The following lemma asserts that the same is true even when the regularity is measured in terms of the ultradifferentiable classes of Section 2. To do so, we consider $u=f \chi$ where $f$ is a (possibly) quasianalytic function and $\chi$ is a cut-off function.

Lemma 3.2. Let $\omega$ be a sublinear weight function as in Definition 2.2. Let $V_{0} \Subset$ $V \subset \mathbb{R}^{n}$ be open sets, $f \in \mathcal{E}_{(\omega)}(V)$, and let $\chi \in C_{0}^{\infty}\left(\mathbb{R}^{n}\right)$ be a differentiable function satisfying $\chi \equiv 1$ on a neighborhood of $\bar{V}_{0}$ and $\operatorname{supp} \chi \subset V$. Let $\Delta_{j} \subset \mathbb{R}^{n}$ be closed proper cones $(j=1, \ldots, J)$ with disjoint interiors satisfying $\dot{\mathbb{R}}^{n}=\bigcup_{j=1}^{J} \Delta_{j}$, and define

$$
F_{j}(z)=\int_{\mathbb{R}^{n}} W\left(z-x, \Delta_{j}\right) f(x) \chi(x) d x
$$

for $j=1, \ldots, J$. Then each $F_{j}(z)$ is a holomorphic function in an infinitesimal wedge of the form $\mathbb{R}^{n}+i \Delta_{j}^{\perp} 0$. More precisely, for any strict subcone $G_{j} \subset \Delta_{j}^{\perp}$, there exists a positive constant $d$ such that $F_{j}(z) \in \mathcal{O}\left(\mathbb{R}^{n}+i G_{j}[d]\right)$. For such choices of $G_{j}$ and $d$, all the derivatives $F_{j}^{(\alpha)}(z):=\partial^{\alpha} F_{j}(z)\left(\alpha \in \mathbb{N}_{0}^{n}\right)$ extend continuously to $\mathbb{R}^{n}+i\left(G_{j}[d] \cup\{0\}\right)$. Therefore we may denote by the same symbol $F_{j}^{(\alpha)}(z)$ the extended function on $\mathbb{R}^{n}+i\left(G_{j}[d] \cup\{0\}\right)$, and by $F_{j}^{(\alpha)}(x)$ its restriction on $\mathbb{R}^{n}$. Furthermore if we restrict the domain of definition from $\mathbb{R}^{n}$ to $V_{0}$, then $F_{j}^{(\alpha)}$ belongs to $\mathcal{E}_{(\omega)}\left(V_{0}\right)$, and for any $\alpha \in \mathbb{N}_{0}^{n}$, the map

$$
G_{j}[d] \cup\{0\} \ni s \mapsto F_{j}^{(\alpha)}(\cdot+i s) \in \mathcal{E}_{(\omega)}\left(V_{0}\right)
$$

is continuous. 
Proof. The statements on the estimates of the domains of holomorphy of $F_{j}$ and the extendibility of $F_{j}^{(\alpha)}$ are consequences of the fact that the $F_{j}$ are the convolutions of $\chi(x) f(x) \in C_{0}^{\infty}\left(\mathbb{R}^{n}\right)$ with the twisted Radon kernel associated with $\Delta_{j}$. The remaining statements follow from the estimate

$$
\begin{aligned}
\exists d>0, \forall \lambda>0, \exists C_{\lambda}>0, \forall j \in\{1, \ldots, J\}, \forall \alpha \in \mathbb{N}_{0}^{n}, \\
\sup _{x \in \bar{V}_{0}, s \in G_{j}[d]}\left|\partial^{\alpha} F_{j}(x+i s)\right| \leq C_{\lambda} \exp \left(\varphi_{[\lambda]}^{*}(|\alpha|)\right) .
\end{aligned}
$$

In fact, assume for the moment that we have proved (3.4). Then, for any $x \in \bar{V}_{0}$, $s \in G_{j}[d], \lambda>0,1 \leq j \leq J$ and $\alpha, \beta \in \mathbb{N}_{0}^{n}$, from Proposition 2.4(e) we have

$$
\left|\partial^{\alpha+\beta} F_{j}(x+i s)\right| \leq C_{2 \lambda} \exp \left(\varphi_{[2 \lambda]}^{*}(|\alpha+\beta|)\right) \leq C_{2 \lambda} \exp \left(\varphi_{[\lambda]}^{*}(|\alpha|)\right) \cdot \exp \left(\varphi_{[\lambda]}^{*}(|\beta|)\right),
$$

which implies

$$
p_{\omega, \lambda, \bar{V}_{0}}\left(F^{(\alpha)}(\cdot+i s)\right) \leq C_{2 \lambda} \exp \left(\varphi_{[\lambda]}^{*}(|\alpha|)\right) .
$$

Moreover, for $s_{1}$ and $s_{2}$ in $G_{j}[d]$,

$$
\begin{aligned}
\left|F_{j}^{(\alpha+\beta)}\left(x+i s_{1}\right)-F_{j}^{(\alpha+\beta)}\left(x+i s_{2}\right)\right| & \leq\left|s_{1}-s_{2}\right| \sum_{k=1}^{n} \sup _{s \in\left[s_{1}, s_{2}\right]}\left|\partial_{z_{k}} F_{j}^{(\alpha+\beta)}(x+i s)\right| \\
& \leq 2 d n C_{2 \lambda} \exp \left(\varphi_{[2 \lambda]}^{*}(|\alpha+\beta|+1)\right),
\end{aligned}
$$

where $\left[s_{1}, s_{2}\right]$ denotes the line segment with endpoints $s_{1}$ and $s_{2}$, and we have used $\left|s_{1}-s_{2}\right| \leq 2 d$. Therefore

$$
p_{\omega, \lambda, \bar{V}_{0}}\left(F^{(\alpha)}\left(\cdot+i s_{1}\right)-F^{(\alpha)}\left(\cdot+i s_{2}\right)\right) \leq 2 d n C_{2 \lambda} \exp \left(\varphi_{[\lambda]}^{*}(|\alpha|+1)\right) .
$$

In this way, we obtain the boundedness and the uniform continuity of the map (3.3) on $G_{j}[d]$.

Now we prove the estimate (3.4). To do so, we start with the following claim.

Claim 3.3. Let $\alpha=\left(\alpha_{1}, \ldots, \alpha_{n}\right) \in \mathbb{N}_{0}^{n}$ and let $k, l$ be integers with $1 \leq k \leq n$ and $1 \leq l \leq \alpha_{k}$. With the notation

$$
\alpha_{<k}=\left(\alpha_{1}, \ldots, \alpha_{k-1}, 0, \ldots, 0\right), \quad \alpha_{>k}=\left(0, \ldots, 0, \alpha_{k+1}, \ldots, \alpha_{n}\right),
$$

we have

$$
\begin{aligned}
& \partial^{\alpha} F_{j}(z) \\
& =\sum_{k=1}^{n} \sum_{1 \leq l \leq \alpha_{k}} \int_{\mathbb{R}^{n}}\left(\partial_{z_{k}}^{\alpha_{k}-l} \partial_{z}^{\alpha_{>k}} W\left(z-x, \Delta_{j}\right)\right)\left(\partial_{x_{k}}^{l-1} \partial_{x}^{\alpha_{<k}} f(x)\right)\left(\partial_{x_{k}} \chi(x)\right) d x \\
& +\int_{\mathbb{R}^{n}} W\left(z-x, \Delta_{j}\right)\left(\partial_{x}^{\alpha} f(x)\right) \chi(x) d x .
\end{aligned}
$$


Proof. This claim follows easily from the following formula of integration by parts. Let $X_{q}(q=1, \ldots, p)$ be vector fields with adjoint operators ${ }^{t} X_{q}$. For $f, g \in C^{p}\left(\mathbb{R}^{n}\right)$ and $\chi \in C_{0}^{1}\left(\mathbb{R}^{n}\right)$, we have (e.g., by induction)

$$
\begin{aligned}
\int_{\mathbb{R}^{n}}\left({ }^{t} X_{1} \cdots{ }^{t} X_{p} g\right) f \chi d x= & \sum_{q=1}^{p} \int_{\mathbb{R}^{n}}\left({ }^{t} X_{q+1} \cdots{ }^{t} X_{p} g\right)\left(X_{q-1} \cdots X_{1} f\right)\left(X_{q} \chi\right) d x \\
& +\int_{\mathbb{R}^{n}} g\left(\left(X_{p} \cdots X_{1}\right) f\right) \chi d x .
\end{aligned}
$$

(Notice that $\int\left({ }^{t} X g\right)(f \chi) d x=\int g((X f) \chi+f X \chi) d x$. The main point in the procedure is that once one of the fields $X_{j}$ falls on $\chi$, we stop integrating by parts. This idea was used by [6] for the estimates of derivatives of convolutions with Gaussian kernels of ultradifferentiable functions multiplied by a cut-off function. Refer to the proof of [6, Proposition 3.2].)

We continue the proof of Lemma 3.2 , denoting by $F_{j, 1, \alpha}(z)$ and by $F_{j, 2, \alpha}(z)$ the first and the second terms on the right hand side of (3.5). Since $f \in \mathcal{E}_{(\omega)}(V)$, there exists for any $\mu>0$ a constant $A_{\mu}>0$ such that

$$
\left|\partial_{x}^{\gamma} f(x)\right| \leq A_{\mu} \exp \left(\varphi_{[\mu]}^{*}(|\gamma|)\right) \quad \text { for } x \in \operatorname{supp} \chi \text { and } \gamma \in \mathbb{N}_{0}^{n} .
$$

Note that the sum $\sum_{k=1}^{n} \sum_{1 \leq l \leq \alpha_{k}}$ of $F_{j, 1, \alpha}$ consists of $|\alpha|$ terms, and it can be written in the form

$$
F_{j, 1, \alpha}(z)=\sum_{q=1}^{p} \int_{K_{1}} W_{\alpha, q}\left(z-x, \Delta_{j}\right) f_{\alpha, q}(x) \chi_{\alpha, q}(x) d x \quad(p=|\alpha|),
$$

where each $W_{\alpha, q}$ (resp. $f_{\alpha, q}, \chi_{\alpha, q}$ ) is one of the derivatives of $W$ (resp. $f, \chi$ ) of order $p-q$ (resp. $q-1,1)$, and $K_{1}:=\bigcup_{k=1}^{n} \operatorname{supp} \partial_{x_{k}} \chi$ is a compact set satisfying $\operatorname{dist}\left(K_{1}, \bar{V}_{0}\right)>0$. Therefore, we have

$$
\left\|\chi_{\alpha, q}\right\|_{L^{1}\left(K_{1}\right)} \leq c_{0}:=\sup _{1 \leq k \leq n}\left\|\partial_{x_{k}} \chi\right\|_{L^{1}\left(K_{1}\right)}<\infty .
$$

Since $W(z, \xi)$ is holomorphic in a neighborhood of $\left(\mathbb{R}^{n} \backslash\{0\}\right) \times S^{n-1}$, and since $\operatorname{dist}\left(K_{1}, \bar{V}_{0}\right)>0$, there exist positive constants $d_{1}, a_{0}$ and $a_{1}$ such that

$$
\left|\partial_{z}^{\beta} W(z-x, \xi)\right| \leq a_{0} a_{1}^{|\beta|}|\beta| !
$$

for $x \in K_{1}$, Re $z \in \bar{V}_{0},|\operatorname{Im} z| \leq d_{1}, \xi \in S^{n-1}, \beta \in \mathbb{N}_{0}^{n}$. Moreover, since $\omega$ is sublinear, we can use Proposition 2.4(h) and take, for any $\mu>0$, a constant $B_{\mu}$ such that

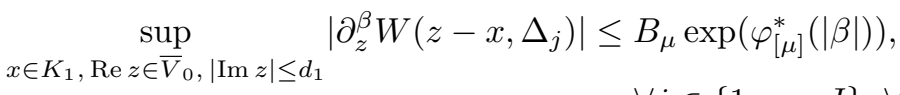

$$
\begin{aligned}
& \forall j \in\{1, \ldots, J\}, \forall \beta \in \mathbb{N}_{0}^{n} .
\end{aligned}
$$


From (3.6)-(3.8), the first term $F_{j, 1, \alpha}(z)$ on the right hand side of $(3.5)$ is estimated, for any $\mu>0$, as

$$
\begin{aligned}
& \left|F_{j, 1, \alpha}(z)\right| \leq \sum_{q=1}^{p} B_{\mu} \exp \left(\varphi_{[\mu]}^{*}(p-q)\right) \cdot A_{\mu} \exp \left(\varphi_{[\mu]}^{*}(q-1)\right) \cdot c_{0} \\
& \quad \leq A_{\mu} B_{\mu} c_{0} \cdot \exp \left(\varphi_{[\mu]}^{*}(p-1)+\log p\right) \quad \text { for } \operatorname{Re} z \in \bar{V}_{0},|\operatorname{Im} z|<d_{1} .
\end{aligned}
$$

Here we have used Proposition 2.4(d) to write $\varphi_{[\mu]}^{*}(p-q)+\varphi_{[\mu]}^{*}(q-1) \leq \varphi_{[\mu]}^{*}(p-1)$, and we have again denoted $p=|\alpha|$. Now for any given $\lambda>0$, we can apply (2.4) in Proposition 2.4(g), and get a suitable $\mu$ and $c$ with $\varphi_{[\lambda]}^{*}(p)-p \geq \varphi_{[\mu]}^{*}(p)-c$. We can use (3.9) for this $\mu$ to get $C_{1, \lambda}$ such that

$$
\left|F_{j, 1, \alpha}(z)\right| \leq C_{1, \lambda} \exp \left(\varphi_{[\lambda]}^{*}(|\alpha|)\right) \quad \text { for } \operatorname{Re} z \in \bar{V}_{0},|\operatorname{Im} z|<d_{1},
$$

and for any $\lambda>0,1 \leq j \leq J, \alpha \in \mathbb{N}_{0}^{n}$.

As for the second term on the right hand side of (3.5), we write

$$
F_{j, 2, \alpha}(z)=\int_{K} d x \int_{S^{n-1} \cap \Delta_{j}} W(z-x, \xi)\left(\partial_{x}^{\alpha} f(x)\right) \chi(x) d \sigma(\xi) \quad\left(K:=\operatorname{supp} \chi \subset \mathbb{R}^{n}\right),
$$

and use the formula

$$
W(z, \xi)=-\frac{1+i\langle z, \xi\rangle}{(2 \pi i)^{n}}\left(\frac{1}{1+2 i\langle z, \xi\rangle}\left\langle\xi, \partial_{z}\right\rangle\right)^{n} \log \Phi(z, \xi)
$$

which follows from $\left\langle\xi, \partial_{z}\right\rangle \Phi(z, \xi)=1+2 i\langle z, \xi\rangle$. Integrating by parts, and using $\left\langle\xi, \partial_{z}\right\rangle(1+i\langle z, \xi\rangle)=i$ and $\left\langle\xi, \partial_{z}\right\rangle(1+2 i\langle z, \xi\rangle)=2 i$, we get

$$
\begin{aligned}
& F_{j, 2, \alpha}(z)= \int_{K} d x \int_{S^{n-1} \cap \Delta_{j}} \log \Phi(z-x, \xi) \sum_{\substack{k \in\{0,1\}, l, m \in \mathbb{N}_{0}, k+l+m=n}} b_{k, l, m} \\
& \times \frac{(1+i\langle z-x, \xi\rangle)^{1-k}}{(1+2 i\langle z-x, \xi\rangle)^{n+l}}\left\langle\xi, \partial_{x}\right\rangle^{m}\left\{\left(\partial_{x}^{\alpha} f(x)\right) \chi(x)\right\} d \sigma(\xi) \\
&= \int_{K} d x \int_{S^{n-1} \cap \Delta_{j}} \log \Phi(z-x, \xi) \sum_{\substack{k \in\{0,1\}, l, m \in \mathbb{N}_{0}, k+l+m=n}} b_{k, l, m} \sum_{\substack{\beta, \gamma \in \mathbb{N}_{0}^{n},|\beta|+|\gamma|=m}} \frac{m !}{\beta ! \gamma !} \xi^{\beta+\gamma} \\
& \times \frac{(1+i\langle z-x, \xi\rangle)^{1-k}}{(1+2 i\langle z-x, \xi\rangle)^{n+l}}\left(\partial_{x}^{\alpha+\beta} f(x)\right)\left(\partial_{x}^{\gamma} \chi(x)\right) d \sigma(\xi) \\
& \int_{K} d x \int_{S^{n-1} \cap \Delta_{j}} \log \Phi(z-x, \xi) \\
& \times \sum_{|\beta|+|\gamma| \leq n} b_{\beta, \gamma}(z-x, \xi)\left(\partial_{x}^{\alpha+\beta} f(x)\right)\left(\partial_{x}^{\gamma} \chi(x)\right) d \sigma(\xi),
\end{aligned}
$$


where $b_{k, l, m}$ are some constants, and $b_{\beta, \gamma}(z, \xi)$ are analytic functions on $\{(z, \xi) \in$ $\left.\mathbb{C}^{n} \times S^{n-1} ;|\operatorname{Im} z|<1 / 2\right\}$. In fact, $(1+2 i\langle z, \xi\rangle)^{2 n} b_{\beta, \gamma}(z, \xi)$ are polynomials in $\langle z, \xi\rangle$ and $\xi$, and $1+2 i\langle z, \xi\rangle$ does not vanish if $|\operatorname{Im} z|<1 / 2$ and $|\xi|=1$. (The exact form of these constants is not needed in the argument, since there are only finitely many of them.)

Therefore, using (3.6), $F_{j, 2, \alpha}(z)$ is estimated as

$$
\begin{aligned}
& \left|F_{j, 2, \alpha}(z)\right| \leq A_{2 \lambda} \exp \left(\varphi_{[2 \lambda]}^{*}(|\alpha|+n)\right)\|\chi\|_{C^{n}(K)} \\
& \times \sum_{|\beta|+|\gamma| \leq n} \sup _{x \in K, \xi \in S^{n-1}}\left|b_{\beta, \gamma}(z-x, \xi)\right| \cdot \int_{K} \sup _{\xi \in S^{n-1} \cap \Delta_{j}}|\log \Phi(z-x, \xi)| d x \\
& \times \int_{S^{n-1} \cap \Delta_{j}} d \sigma(\xi) \quad \text { for }|\operatorname{Im} z|<1 / 2,
\end{aligned}
$$

and for any $\lambda>0,1 \leq j \leq J, \alpha \in \mathbb{N}_{0}^{n}$.

By Proposition 2.4(e), we have

$$
A_{2 \lambda} \exp \left(\varphi_{[2 \lambda]}^{*}(|\alpha|+n)\right) \leq A_{2 \lambda} \exp \left(\varphi_{[\lambda]}^{*}(n)\right) \cdot \exp \left(\varphi_{[\lambda]}^{*}(|\alpha|)\right) .
$$

Since $G_{j}$ is a strict subcone of $\Delta_{j}^{\perp}$, we have $\inf _{\xi \in S^{n-1} \cap \Delta_{j}, s \in G_{j}}\langle s /|s|, \xi\rangle>0$, and therefore we can take a constant $d_{2}$ with

$$
0<d_{2}<\min \left\{1 / 2, \min _{1 \leq j \leq J} \inf _{\xi \in S^{n-1} \cap \Delta_{j}, s \in G_{j}}\langle s /|s|, \xi\rangle\right\} .
$$

Since $d_{2}<1 / 2$, we have

$$
c_{1}:=\sup _{z \in \bar{V}_{0}+i G_{j}\left[d_{2}\right]} \sum_{|\beta|+|\gamma| \leq n} \sup _{x \in K, \xi \in S^{n-1}}\left|b_{\beta, \gamma}(z-x, \xi)\right|<\infty .
$$

On the other hand, since $0<d_{2}<\inf _{\xi \in S^{n-1} \cap \Delta_{j}, s \in G_{j}}\langle s /|s|, \xi\rangle$ for any $j$, we have $\langle s, \xi\rangle-|s|^{2} \geq 0$ for $s \in G_{j}\left[d_{2}\right]$. Using also $\operatorname{Im} \Phi(z-x, \xi)=\langle\operatorname{Im} z, \xi\rangle-|\operatorname{Im} z|^{2}+$ $|\operatorname{Re} z-x|^{2}$, we get the estimates

$$
\operatorname{Im} \log \Phi(z-x, \xi) \in[0, \pi],
$$

$\operatorname{Re} \log \Phi(z-x, \xi) \geq \log \operatorname{Im} \Phi(z-x, \xi) \geq 2 \log |\operatorname{Re} z-x|$,

for $z \in \bar{V}_{0}+i G_{j}\left[d_{2}\right]$, and therefore

$$
c_{2}:=\sup _{z \in \bar{V}_{0}+i G_{j}\left[d_{2}\right]} \int_{K} \sup _{\xi \in S^{n-1} \cap \Delta_{j}}|\log \Phi(z-x, \xi)| d x<\infty .
$$

Finally, from (3.11)-(3.14) we get

$$
\left|F_{j, 2, \alpha}(z)\right| \leq C_{2, \lambda} \exp \left(\varphi_{[\lambda]}^{*}(|\alpha|)\right) \quad \text { for } z \in \bar{V}_{0}+i G_{j}\left[d_{2}\right],
$$


and for any $\lambda>0,1 \leq j \leq J, \alpha \in \mathbb{N}_{0}^{n}$, where $C_{2, \lambda}:=c_{1} c_{2}\left|S^{n-1}\right| \cdot\|\chi\|_{C^{n}(K)} \cdot$ $A_{2 \lambda} \exp \left(\varphi_{[\lambda]}^{*}(n)\right)$.

By defining $d:=\min \left\{d_{1}, d_{2}\right\}$, the estimates (3.10) and (3.15) give (3.4), which concludes the proof of Lemma 3.2.

Theorem 3.4. Let $V \times U \subset \mathbb{R}^{n} \times \mathbb{R}^{m}$ be an open set and consider $\mathcal{K} \in \mathcal{E}_{(\omega)}(V \times U)$ satisfying (1.1). Then, for any $V_{0} \times U_{0} \Subset V \times U, \mathcal{K}$ admits, on $V_{0} \times U_{0}$, a boundary value representation

$$
\mathcal{K}(x, y)=\sum_{j=0}^{J} b_{\hat{G}_{j}}\left(F_{j}(z, w)\right),
$$

where $\hat{G}_{j} \subset \mathbb{R}^{n+m}(j=0,1, \ldots, J)$ are open convex cones which include $G_{j} \times\{0\}$ for some open convex cones $G_{j} \subset \mathbb{R}^{n}$, the $F_{j}$ belong to $\mathcal{O}\left(V_{0} \times U_{0}+i \hat{G}_{j}[d]\right) \cap$ $C\left(V_{0} \times U_{0}+i\left(\hat{G}_{j}[d] \cup\{0\}\right)\right)$ with some $d>0$, such that for each fixed $z \in$ $V_{0}+i\left(G_{j}[d] \cup\{0\}\right)$, the map $U_{0} \ni y \mapsto F_{j}(z, y)$ is an ultradifferentiable function in $\mathcal{E}_{(\omega)}\left(U_{0}\right)$ even in the case $z \in V_{0}$ (which means in particular that $z$ is real), and that for any $\alpha \in \mathbb{N}_{0}^{n}$ and any $j$, the correspondence

$$
V_{0}+i\left(G_{j}[d] \cup\{0\}\right) \ni z \mapsto \partial_{x}^{\alpha} F_{j}(z, \cdot) \in \mathcal{E}_{(\omega)}\left(U_{0}\right)
$$

is continuous. In particular,

$$
\mathcal{K}(x, y)=\sum_{j=0}^{J} F_{j}(x, y) .
$$

Proof. We take a cut-off function $\chi \in C_{0}^{\infty}(V \times U)$ with $\chi \equiv 1$ in a neighborhood of $\overline{V_{0} \times U_{0}}$. We can take a decomposition $\mathbb{R}^{n+m}=\bigcup_{j=1}^{J} \Delta_{j}$ into proper closed convex cones $\Delta_{j} \subset \dot{\mathbb{R}}^{n+m}$ with disjoint interiors and a number $J_{0}<J$ such that

$$
\begin{array}{ll}
\Delta_{j}^{\perp} \cap\left(\dot{\mathbb{R}}^{n} \times\{0\}\right) \neq \emptyset & \left(j \leq J_{0}\right), \\
\left(V_{0} \times U_{0} \times \Delta_{j}\right) \cap \mathrm{WF}_{A} \mathcal{K}=\emptyset & \left(j>J_{0}\right) .
\end{array}
$$

In fact, since $\mathcal{K}$ satisfies (1.1) and since $V_{0} \times U_{0} \Subset V \times U$, there exists a constant $\varepsilon>0$ satisfying

$$
\mathrm{WF}_{A} \mathcal{K} \cap\left\{(x, y ; \xi, \eta) ;(x, y) \in \overline{V_{0} \times U_{0}},|\xi| \leq 2 \varepsilon|\eta|\right\}=\emptyset .
$$

Then we may construct a covering $\mathbb{R}^{n+m}=\bigcup_{j} \Delta_{j}$ consisting of proper closed convex cones $\Delta_{j}$ with disjoint interiors, satisfying the additional conditions

$$
\bigcup_{j=1}^{J_{0}} \Delta_{j} \subset\{|\xi|>\varepsilon|\eta|\}, \quad \bigcup_{j=J_{0}+1}^{J} \Delta_{j} \subset\{|\xi|<2 \varepsilon|\eta|\} .
$$

Then for $j \leq J_{0}, \Delta_{j} \cap\left(\{0\} \times \mathbb{R}^{m}\right)=\emptyset$ implies (3.17), and (3.18) is trivially satisfied. 
Now we apply Lemma 3.2 to the data $V_{0} \times U_{0} \Subset V \times U \subset \mathbb{R}^{n+m}, \mathcal{K} \in$ $\mathcal{E}_{(\omega)}(V \times U), \chi \in C_{0}^{\infty}\left(\mathbb{R}^{n+m}\right)$ and $\Delta_{j} \subset \dot{\mathbb{R}}^{n+m}$, and get holomorphic functions $F_{j}(z, w)$ on infinitesimal wedges of the form $\mathbb{R}^{n}+i \Delta_{j}^{\perp} 0$.

Let us first consider $j=1, \ldots, J_{0}$. For these $j$, we can take cones $G_{j} \subset \mathbb{R}^{n}$ and strict subcones $\hat{G}_{j} \subset \mathbb{R}^{n+m}$ of $\Delta_{j}^{\perp}$ such that $G_{j} \times\{0\} \subset \hat{G}_{j}$, since the $\Delta_{j}$ satisfy (3.17). Moreover, when $z=x+i s \in V_{0}+i\left(G_{j}[d] \cup\{0\}\right)$ is fixed, the function $U_{0} \ni$ $y \mapsto \partial_{x}^{\alpha} F_{j}(z, y)$ is a restriction of the function $V_{0} \times U_{0} \ni(x, y) \mapsto \partial_{x}^{\alpha} F_{j}((x, y)+$ $i(s, 0))$ with respect to the $x$ variable. Therefore, it belongs to $\mathcal{E}_{(\omega)}\left(U_{0}\right)$. In addition, the continuity of

$$
G_{j}[d] \cup\{0\} \ni s \mapsto \partial_{x}^{\alpha} F_{j}(\cdot+i s, \cdot) \in \mathcal{E}_{(\omega)}\left(V_{0} \times U_{0}\right)
$$

(coming from the continuity of (3.3)) implies the locally uniform continuity of

$$
G_{j}[d] \cup\{0\} \ni s \mapsto \partial_{x}^{\alpha} F_{j}(x+i s, \cdot) \in \mathcal{E}_{(\omega)}\left(U_{0}\right)
$$

in $x \in V_{0}$, which also implies the continuity of (3.16).

Next we consider $j=J_{0}+1, \ldots, J$. In view of (3.18), we can apply Theorem 3.1 to $V_{0} \times U_{0} \Subset V \times U \subset \mathbb{R}^{n+m}, \chi(x, y) \mathcal{K}(x, y)$ and $\Delta_{j} \subset \dot{\mathbb{R}}^{n+m}$. Since $F_{j}$ is realanalytic in a neighborhood of $\overline{V_{0} \times U_{0}}$, we can ignore $\Delta_{j}$ and take $\hat{G}_{j}=\mathbb{R}^{n+m}$ and $G_{j}=\mathbb{R}^{n}$. Then the map

$$
V_{0}+i\{s ;|s|<d\} \ni z \mapsto \partial_{x}^{\alpha} F_{j}(z, \cdot) \in \mathcal{A}\left(U_{0}\right)
$$

is well-defined and the usual Cauchy estimate proves its continuity. The continuity of the canonical embedding $\mathcal{A}\left(U_{0}\right) \hookrightarrow \mathcal{E}_{(\omega)}\left(U_{0}\right)$ gives the desired estimate for these $j$.

In this way we have proved the continuity of (3.16) for both cases, which concludes the proof of Theorem 3.4.

\section{$\S 4$. Compatibility questions related to the definition of $T$}

We discuss here the integral operator $u \mapsto \int \mathcal{K}(x, y) u(y) d y$ of (1.2) in the special case when $\mathcal{K} \in \mathcal{E}_{(\omega)}(V \times U)$ (still satisfying (1.1)) and $u \in \mathcal{E}_{(\omega)}^{\prime}(U)$. We recall that initially in (1.2) the integration $\int \mathcal{K}(x, y) u(y) d y$ is the one along fibers in the theory of hyperfunctions, but we have already rewritten it in $[12,(4.3)]$ as an explicit formula in terms of holomorphic defining functions for the kernel. It will be convenient to take for these defining functions the ones given by Theorem 3.4. We can then in fact give a new interpretation for our integrals using the duality between ultradifferentiable functions and the corresponding functionals.

In this section, we study the compatibility between the two interpretations so obtained for (1.2) in our special case. We refer to [12, Remark 6.2(ii)] for a similar compatibility question in the case of differentiable kernels and Radon measures. As a corollary to the results in Section 3 we can in fact prove 
Corollary 4.1. Let $\omega$ be a weight function and let $V \times U \subset \mathbb{R}^{n} \times \mathbb{R}^{m}$ be an open set. Then for any $\mathcal{K} \in \mathcal{E}_{(\omega)}(V \times U)$ satisfying (1.1) and any $u \in \mathcal{E}_{(\omega)}^{\prime}(U)$, we have

$$
\int_{U} \mathcal{K}(x, y) u(y) d y=\langle u, \mathcal{K}(x, \cdot)\rangle .
$$

Here the left hand side is the integration of the hyperfunction $\mathcal{K} u$ with $u$ regarded as a hyperfunction on $U$ with compact support, and the right hand side is a continuous function on $V$ whose value for each fixed $x$ is computed by the duality bilinear form $\mathcal{E}_{(\omega)}^{\prime}(U) \times \mathcal{E}_{(\omega)}(U) \rightarrow \mathbb{C}$, and is regarded as a hyperfunction on $V$.

Note that $\mathcal{K} u$ as a product of hyperfunctions is well-defined because of the wave front set estimate (1.1), and also that the restriction $y \mapsto \mathcal{K}(x, y)$ at each fixed $x$ belongs to $\mathcal{E}_{(\omega)}(U)$ in a trivial way.

Proof. It suffices to prove the equality on each relatively compact open set $V_{0} \Subset V$. We take a relatively compact open set $U_{0} \Subset U$ which includes supp $u$. Applying Theorem 3.4 to $\mathcal{K}$ and $V_{0} \times U_{0}$, we get a representation $\mathcal{K}(x, y)=$ $\sum_{j=0}^{J} b_{\hat{G}_{j}}\left(F_{j}(z, w)\right)$ on $V_{0} \times U_{0}$ satisfying the additional properties stated in the theorem. In view of $[12,(4.3)]$, this representation enables us to calculate the left hand side of (4.1) as

$$
\int_{U} \mathcal{K}(x, y) u(y) d y=\sum_{j=0}^{J} b_{G_{j}}\left(f_{j}(z)\right),
$$

where each $f_{j}$ is a holomorphic function on $V_{0}+i G_{j}[d]$ given by

$$
f_{j}(z):=\int_{U_{0}} F_{j}(z, y) u(y) d y=\left\langle u, F_{j}(z, \cdot)\right\rangle \quad \text { for } z \in V_{0}+i G_{j}[d] .
$$

On the other hand, it follows, again from Theorem 3.4, that the right hand side of (4.2) defines a continuous function on $V_{0}+i\left(G_{j}[d] \cup\{0\}\right)$, since $z \mapsto F_{j}(z, \cdot) \in$ $\mathcal{E}_{(\omega)}\left(U_{0}\right)$ is continuous on that set. Therefore each $f_{j}(z)$ admits a boundary value in $\mathcal{E}_{(\omega)}\left(U_{0}\right)$ and we have

$$
b_{G_{j}}\left(f_{j}(z)\right)=\left\langle u, F_{j}(x, \cdot)\right\rangle .
$$

We conclude that

$$
\begin{aligned}
\int_{U} \mathcal{K}(x, y) u(y) d y & =\sum_{j=0}^{J} b_{G_{j}}\left(f_{j}(z)\right)=\sum_{j=0}^{J}\left\langle u, F_{j}(x, \cdot)\right\rangle \\
& =\left\langle u, \sum_{j=0}^{J} F_{j}(x, \cdot)\right\rangle=\langle u, \mathcal{K}(x, \cdot)\rangle
\end{aligned}
$$

as hyperfunctions on $V_{0}$. 


\section{§5. Division of the kernel}

The following result will play the role of [12, Theorem 5.3], but is here adapted to an ultradifferentiable setting.

Theorem 5.1. Let $\mathcal{K} \in \mathcal{B}(V \times U)$ be a hyperfunction satisfying the wave front set estimate (1.1), and $\omega$ a sublinear weight function. Then there exist an elliptic (possibly infinite order) partial differential operator $P\left(D_{x}\right)$ with constant coefficients in the variable $x$, a function $\mathcal{K}^{\prime} \in \mathcal{E}_{(\omega)}(V \times U)$, which also satisfies the wave front set estimate

$$
\left\{(x, y ; 0, \eta) \in V \times U \times \mathbb{R}^{n} \times \mathbb{R}^{m} ; \eta \neq 0\right\} \cap \mathrm{WF}_{A} \mathcal{K}^{\prime}=\emptyset,
$$

and a real-analytic function $\mathcal{K}^{\prime \prime}$ on $V \times U$ such that $\mathcal{K}=P\left(D_{x}\right) \mathcal{K}^{\prime}+\mathcal{K}^{\prime \prime}$.

As a preparation for the proof of Theorem 5.1, we start with the following lemma.

Lemma 5.2. Let $\omega$ be a weight function. For a measurable function $\mu(\xi)$ on $\mathbb{R}^{n}$ satisfying

$$
\mu(\xi) \exp (\lambda \omega(|\xi|)) \in L^{1}\left(\mathbb{R}^{n}\right) \quad \text { for any } \lambda>0,
$$

the inverse Fourier transform $\left(\mathcal{F}^{-1} \mu\right)(x)$ belongs to $\mathcal{E}_{(\omega)}\left(\mathbb{R}^{n}\right)$.

Proof. We have, for any $\alpha \in \mathbb{N}_{0}^{n}$ and any $\lambda>0$,

$$
\begin{aligned}
\left\|\xi^{\alpha} \mu(\xi)\right\|_{L^{1}\left(\mathbb{R}^{n}\right)} & \leq\left\||\xi|^{|\alpha|} e^{-\lambda \omega(|\xi|)}\right\|_{L^{\infty}\left(\mathbb{R}^{n}\right)} \cdot\left\|\mu(\xi) e^{\lambda \omega(|\xi|)}\right\|_{L^{1}\left(\mathbb{R}^{n}\right)} \\
& \leq \exp \left(\varphi_{[\lambda]}^{*}(|\alpha|)\right) \cdot\left\|\mu(\xi) e^{\lambda \omega(|\xi|)}\right\|_{L^{1}\left(\mathbb{R}^{n}\right) .}
\end{aligned}
$$

Therefore $\mathcal{F}^{-1} \mu$ is differentiable, and satisfies

$$
p_{\omega, \lambda, K}\left(\mathcal{F}^{-1} \mu\right) \leq(2 \pi)^{-n}\left\|\mu(\xi) e^{\lambda \omega(|\xi|)}\right\|_{L^{1}\left(\mathbb{R}^{n}\right)}
$$

for any $K \Subset \mathbb{R}^{n}$.

Proof of Theorem 5.1. The proof is similar to that of [12, Theorem 5.3]. In order to explain the differences in the argument, we recall now the steps in the proof of that theorem and mention how they have to be adapted to our present needs.

Step 1. We first take compact subsets $K_{1} \Subset K_{2} \Subset \cdots$ in $V \times U$ with $\bigcup_{j} K_{j}=$ $V \times U$, hyperfunctions $\mathcal{K}_{j} \in \mathcal{B}\left(\mathbb{R}^{n+m}\right)$ with compact support each of which coincides with $\mathcal{K}$ in a neighborhood of $K_{j}$, positive constants $c_{j}$ and sublinear functions $\ell_{j}$ such that

$$
\begin{gathered}
\mathrm{WF}_{A} \mathcal{K}_{j} \cap\left\{(x, y ; \xi, \eta) ;(x, y) \in K_{j}\right\} \subset\left\{(x, y ; \xi, \eta) ;|\eta| \leq c_{j}|\xi|\right\}, \\
\left|\mathcal{F} \mathcal{K}_{j}(\xi, \eta)\right| \leq \exp \ell_{j}(\xi, \eta) \quad \text { for any }(\xi, \eta) \in \mathbb{R}^{n+m} .
\end{gathered}
$$


Next we take a function $\tilde{\omega}(t)$ satisfying $\omega(t)=o(\tilde{\omega}(t))$ and $\tilde{\omega}(t)=o(t)$ as $t \rightarrow \infty$, define sublinear functions $\tilde{\ell}_{j}$ by

$$
\tilde{\ell}_{j}(\xi):=\sup _{|\eta| \leq 2 c_{j}|\xi|}\left(\ell_{j}(\xi, \eta)+|(\xi, \eta)|^{1 / 2}+\tilde{\omega}(|\xi|)\right),
$$

and take a sublinear function $\ell$ and constants $c_{j}^{\prime}$ such that $\tilde{\ell}_{j}(\xi) \leq \ell(\xi)+c_{j}^{\prime}$ for any $\xi \in \mathbb{R}^{n}$ and $j$ (see e.g. [12, Lemma 5.1]). The formula (5.2) replaces [12, (5.7)], where however no $\tilde{\omega}$ was present. We then get the estimate

$$
\left|\mathcal{F} \mathcal{K}_{j}(\xi, \eta)\right| \leq A_{j} \exp \left(\ell(\xi)-|(\xi, \eta)|^{1 / 2}-\tilde{\omega}(|\xi|)\right) \quad \text { if }|\eta| \leq 2 c_{j}|\xi|,
$$

with constants $A_{j}:=e^{c^{\prime}}$. Associated with this $\ell$, we take an infra-exponential elliptic symbol $P(\zeta)\left(\zeta \in \mathbb{C}^{n}\right)$ and $c>0$ satisfying

$$
|P(\zeta)| \geq e^{\ell(\operatorname{Re} \zeta)} \quad \text { if }|\operatorname{Im} \zeta|<c|\operatorname{Re} \zeta|
$$

(see Kawai [11], Kaneko [7]).

Step 2. We define measurable functions $Q_{j}(\xi, \eta)$ by

$$
Q_{j}(\xi, \eta)= \begin{cases}\mathcal{F} \mathcal{K}_{j}(\xi, \eta) / P(\xi) & \text { if }|\eta| \leq 2 c_{j}|\xi| \\ 0 & \text { if }|\eta|>2 c_{j}|\xi|\end{cases}
$$

They satisfy

$$
\begin{gathered}
\operatorname{supp} Q_{j} \subset\left\{|\eta| \leq 2 c_{j}|\xi|\right\} \\
\left|Q_{j}(\xi, \eta)\right| \leq A_{j} \exp \left(-|(\xi, \eta)|^{1 / 2}-\tilde{\omega}(|\xi|)\right) \quad \text { for }(\xi, \eta) \in \mathbb{R}^{n+m}
\end{gathered}
$$

(This corresponds to $[12,(5.9)]$.$) The estimate (5.3) implies \mathcal{F}^{-1} Q_{j} \in \mathcal{E}_{(\omega)}\left(\mathbb{R}^{n+m}\right)$ in view of Lemma 5.2. Now we define the function $\mathcal{K}_{j}^{\prime}$ on $\operatorname{Int} K_{j}$ as the restriction to Int $K_{j}$ of the iterated integral

$$
\int_{|\eta| \leq \frac{3}{2} c_{j}|\xi|} d \sigma(\xi, \eta) \int_{\mathbb{R}^{n+m}} W(x-\tilde{x}, y-\tilde{y}, \xi, \eta)\left(\chi \cdot \mathcal{F}^{-1} Q_{j}\right)(\tilde{x}, \tilde{y}) d \tilde{x} d \tilde{y}
$$

with some cut-off function $\chi \in C_{0}^{\infty}\left(\mathbb{R}^{n+m}\right)$ satisfying $\chi \equiv 1$ on $K_{j}$, and it follows from Lemma 3.2 that $\mathcal{K}_{j}^{\prime} \in \mathcal{E}_{(\omega)}\left(\operatorname{Int} K_{j}\right)$. On the other hand, the estimates of analytic wave front sets as in [12, proof of Theorem 5.3, Step 2] show also in this case that

$$
\mathrm{WF}_{A} \mathcal{K}_{j}^{\prime} \subset\left\{(x, y ; \xi, \eta) ;|\eta| \leq c_{j}|\xi|\right\}, \quad \mathcal{K}_{j}-P\left(D_{x}\right) \mathcal{K}_{j}^{\prime} \in \mathcal{A}\left(\operatorname{Int} K_{j}\right) .
$$

Step 3. Again, it follows from the argument used in [12, proof of Theorem 5.3, Step 3] that $\mathcal{K}_{j}^{\prime}-\mathcal{K}_{k}^{\prime} \in \mathcal{A}\left(\operatorname{Int} K_{j}\right)$ for any $j<k$, and therefore we can take 
$\mathcal{L}_{j} \in \mathcal{A}\left(\operatorname{Int} K_{j}\right)$ with $\mathcal{K}_{j}^{\prime}-\mathcal{K}_{k}^{\prime}=\mathcal{L}_{j}-\mathcal{L}_{k}$ for any $j<k$. Since each $\mathcal{K}_{j}^{\prime}$ belongs to $\mathcal{E}_{(\omega)}\left(\right.$ Int $\left.K_{j}\right)$, so does $\mathcal{K}_{j}^{\prime}-\mathcal{L}_{j}$, and the family $\left(\mathcal{K}_{j}^{\prime}-\mathcal{L}_{j}\right)_{j}$ defines a global section $\mathcal{K}^{\prime} \in \mathcal{E}_{(\omega)}(V \times U)$. We can also show, arguing as in [12, loc.cit.], that $\mathcal{K}^{\prime \prime}:=$ $\mathcal{K}-P\left(D_{x}\right) \mathcal{K}^{\prime}$ is real-analytic. The operator $P\left(D_{x}\right)$ and the functions $\mathcal{K}^{\prime}$ and $\mathcal{K}^{\prime \prime}$ have all the desired properties.

\section{§6. Proof of Theorem 1.1}

We first prove the following intermediate result.

Proposition 6.1. Let $T$ and $\mathcal{K}$ be as in Theorem 1.1, $K \Subset U$ a compact convex set, and let $\ell: \mathbb{C}^{n} \rightarrow \mathbb{R}_{+}$be a sublinear function. Then there exists $L \Subset V$ such that $u \in \mathcal{A}_{\ell}^{\prime}(K)$ implies $\operatorname{singsupp}_{A} T u \subset L$. Here $\mathcal{A}_{\ell}^{\prime}(K)$ denotes the Banach space

$$
\mathcal{A}_{\ell}^{\prime}(K):=\left\{u \in \mathcal{A}^{\prime}(K) ; \sup _{\eta \in \mathbb{C}^{n}}|\hat{u}(\eta)| \exp \left(-H_{K}(\operatorname{Im} \eta)-\ell(\eta)\right)<\infty\right\} .
$$

We also quote the following theorem (see [12, Theorem 6.3]).

Theorem 6.2. Let $T: X \rightarrow C^{\infty}(V)$ be a continuous linear map from some Fréchet space $X$ to $C^{\infty}(V)$. Assume that for every $u \in X$, $\operatorname{singsupp}_{A} T u \subset V$ is compact. Then there exists a compact set $L \subset V$ such that $\operatorname{singsupp}_{A} T u \subset L$ for any $u \in X$

If $T$ were a continuous linear map from $\mathcal{A}_{\ell}^{\prime}(K)$ into $C^{\infty}(V)$ for any $\ell$, then Proposition 6.1 would be an easy consequence of Theorem 6.2 , but in the general case we cannot expect to have $T\left(\mathcal{A}_{\ell}^{\prime}(K)\right) \subset C^{\infty}(V)$. For this reason we use at first the division result of Theorem 5.1 for the kernels.

Proof of Proposition 6.1. We can take a sublinear weight function $\omega(t)$ with $\ell(t)=o(\omega(t))$ as $t \rightarrow \infty$ using Lemma 2.8. By applying Theorem 5.1, we have a decomposition

$$
\mathcal{K}(x, y)=P\left(D_{x}\right) \mathcal{K}^{\prime}(x, y)+\mathcal{K}^{\prime \prime}(x, y),
$$

where $P\left(D_{x}\right)$ is elliptic, $\mathcal{K}^{\prime} \in \mathcal{E}_{(\omega)}(V \times U)$ satisfies the wave front set estimate (5.1), and $\mathcal{K}^{\prime \prime} \in \mathcal{A}(V \times U)$. With the notation $\left(T^{\prime} u\right)(x)=\int_{U} \mathcal{K}^{\prime}(x, y) u(y) d y$, we have $\operatorname{singsupp}_{A} T u=\operatorname{singsupp}_{A} T^{\prime} u$ as pointed out in [12, Remark 5.4]. Therefore, it suffices to prove the present proposition for $T^{\prime}$ instead of $T$, which means that we may assume from the beginning that $\mathcal{K} \in \mathcal{E}_{(\omega)}(V \times U)$.

It can be seen from Definition 2.6, (6.1), and the estimate $\ell(t)=o(\omega(t))$, that $\mathcal{F}\left(\mathcal{A}_{\ell}^{\prime}(K)\right)$ is continuously embedded into $A_{(\omega)}\left(\mathbb{C}^{n}, U\right)$. Therefore Theorem 2.7 implies that the embedding $\mathcal{A}_{\ell}^{\prime}(K) \hookrightarrow \mathcal{E}_{(\omega)}^{\prime}(U)$ is also continuous. 
Moreover, since $\partial_{x}^{\alpha} \mathcal{K} \in \mathcal{E}_{(\omega)}(V \times U)$ for any $\alpha \in \mathbb{N}_{0}^{n}$, Corollary 4.1 yields

$$
\partial_{x}^{\alpha}(T u)(x)=\int_{U} \partial_{x}^{\alpha} \mathcal{K}(x, y) u(y) d y=\left\langle u, \partial_{x}^{\alpha} \mathcal{K}(x, \cdot)\right\rangle \quad \text { for } u \in \mathcal{E}_{(\omega)}^{\prime}(U) .
$$

Therefore $T$ induces a continuous linear map from $\mathcal{E}_{(\omega)}^{\prime}(U)$ to $C^{\infty}(V)$.

Combining these facts, we conclude that $T: \mathcal{A}_{\ell}^{\prime}(K) \rightarrow C^{\infty}(V)$ is a continuous linear map. Since $\mathcal{A}_{\ell}^{\prime}(K)$ is a Banach space and $\operatorname{singsupp}_{A} T u$ is compact for every $u \in \mathcal{A}_{\ell}^{\prime}(K)$, we can now finish the proof by applying Theorem 6.2.

Theorem 6.3. Let $T$ and $\mathcal{K}$ be as in Theorem 1.1. Then for any $K \Subset U$, there exists $L \Subset V$ such that $u \in \mathcal{A}^{\prime}(K)$ implies $\operatorname{singsupp}_{A} T u \subset L$.

Remark 6.4. The main difference between Proposition 6.1 and Theorem 6.3 is of course that, in the proposition, the compact set $L$ in the conclusion might, in principle, depend on $\ell$. By using $\bigcup_{\ell} \mathcal{A}_{\ell}^{\prime}(K)=\mathcal{A}^{\prime}(K)$, and by availing ourselves of the fact that the set of sublinear functions is very big, we shall now show that $L$ can be chosen independently of $\ell$.

Proof of Theorem 6.3. First consider the case when $K$ is convex. Fix $T$ and $\mathcal{K}$, and then take an increasing sequence $L_{1} \subset L_{2} \subset \cdots$ of compact subsets of $V$ with

$$
\bigcup_{j} \operatorname{Int} L_{j}=V
$$

Assume for contradiction that there is no $L \Subset V$ for which $u \in \mathcal{A}^{\prime}(K)$ implies $\operatorname{singsupp}_{A} T u \subset L$. Then, for each $j$, we can find $u_{j} \in \mathcal{A}^{\prime}(K)$ with $\operatorname{singsupp}_{A} T u_{j}$ $\not \subset L_{j}$. Each $u_{j}$ belongs to $\mathcal{A}_{\ell_{j}}^{\prime}(K)$ with some sublinear $\ell_{j}$, and we take a sublinear function $\ell$ and constants $c_{j}^{\prime}$ such that $\ell_{j}(\eta) \leq \ell(\eta)+c_{j}^{\prime}$ for any $\eta \in \mathbb{R}^{n}$ and $j$ (see again e.g. [12, Lemma 5.1]). The latter estimate yields $\mathcal{A}_{\ell_{j}}^{\prime}(K) \subset \mathcal{A}_{\ell}^{\prime}(K)$, therefore all the $u_{j}$ belong to $\mathcal{A}_{\ell}^{\prime}(K)$. Let now $L \subset V$ be a compact set associated with $\ell$ by Proposition 6.1. Then $\operatorname{singsupp}_{A} T u_{j} \subset L$ for any $j$. Moreover, by (6.3), there exists $j^{0}$ satisfying $L \subset \operatorname{Int} L_{j^{0}}$ and in particular $\operatorname{singsupp}_{A} T u_{j} \subset L_{j^{0}}$ for any $j$. This contradicts the choice of the $u_{j}$.

Next we consider the general case, when $K$ is not necessarily convex. We start with a finite covering $K \subset \bigcup_{j=1}^{k} K_{j}$ consisting of convex compact sets $K_{j} \subset U$, and take $L_{j} \Subset V$ for each $j$ such that $u \in \mathcal{A}^{\prime}\left(K_{j}\right)$ implies $\operatorname{singsupp}_{A} T u \subset L_{j}$. Then we can use the flabbiness of $\mathcal{B}$ to obtain $\mathcal{A}^{\prime}(K) \subset \sum_{j} \mathcal{A}^{\prime}\left(K_{j}\right)$. Therefore $L:=\bigcup_{j} L_{j}$ is a compact set with the properties required for $K$.

Proof of Theorem 1.1. We shall show that for any compact set $K \Subset U$, there exists a compact set $L \Subset V$ such that $\operatorname{singsupp}_{A} \mathcal{K} \cap(V \times K) \subset L \times K$. 
Once $K$ is fixed, we consider a second compact set $\tilde{K}$ with $K \Subset \tilde{K} \Subset U$ and associate with it a compact set $L \Subset V$ as in Theorem 6.3 , that is, $u \in \mathcal{A}^{\prime}(\tilde{K})$ must

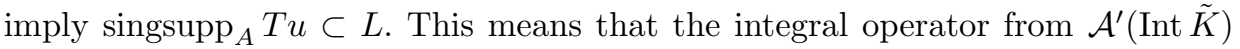
to $\mathcal{B}(V \backslash L)$ associated with the kernel $\left.\mathcal{K}\right|_{(V \backslash L) \times \operatorname{Int} \tilde{K}}$ satisfies the assumptions of Theorem 1.3. Therefore, $\left.\mathcal{K}\right|_{(V \backslash L) \times \operatorname{Int} \tilde{K}}$ is real-analytic and $\operatorname{singsupp}_{A} \mathcal{K} \cap(V \times K) \subset$ $L \times K$.

Remark 6.5. We can now make some comments on why it seemed necessary in this paper to use results from the theory of ultradifferentiable functions and their duals, as opposed to the situation in [12]. Actually, in both papers, a key ingredient is Theorem 6.2 which is applied to an operator of type $T^{\prime} u=\int_{U} \mathcal{K}^{\prime}(x, y) u(y) d y$, where $\mathcal{K}^{\prime}$ is related to $\mathcal{K}$ by a division with remainder of the form (6.2). There are however differences in the way this idea can be implemented. In [12] we could show that $T^{\prime} \equiv 0$ in some region if $T^{\prime} u=0$ there for every Radon measure $u$, by applying the rather strong uniqueness theorem [12, Theorem 1.6] for integral operators of the form (1.2). In the present situation, there is no reasonable counterpart to [12, Theorem 1.6] to test real-analyticity by applying $T^{\prime}$ only to Radon measures, and we had to use Theorem 1.3 instead. It seemed impossible to find a decomposition of type (6.2) which works well simultaneously for all $u \in \mathcal{A}^{\prime}(K)$, but as we have explained, it is possible to find a suitable decomposition which works well for all $u \in \mathcal{A}_{\ell}^{\prime}(K)$ for a fixed sublinear function $\ell$. In fact, a decomposition which is good enough for the argument is when $\mathcal{K}^{\prime}$ is in a BMT class for some weight function $\omega$ related to $\ell$ as above.

We mention incidentally that the reason why there is no full analogue to $[12$, Theorem 1.6] for real-analyticity is discussed in [14, Lemma 2.6 and Example 2.7].

\section{Acknowledgements}

The second author was supported in part by JSPS Grant-in-Aid Nos. 22540173 and 23540186 .

\section{References}

[1] T. Aoki, K. Kataoka, and S. Yamazaki, Hyperfunctions, FBI Transformation, Pseudodifferential Operators of Infinite Order, Kyoritsu, Tokyo, 2004 (in Japanese).

[2] F. Bastin and P. Laubin, On the functional characterization of the analytic wave front set of an hyperfunction, Math. Nachr. 166 (1994), 263-271. Z Zbl 0867.46034 MR 1273337

[3] A general functional characterization of the microlocal singularities, J. Math. Sci. Univ. Tokyo 2 (1995), 155-164. Zbl 0839.58050 MR 1348026

[4] J. Bonet, R. Meise, and S. N. Melikhov, A comparison of two different ways to define classes of ultradifferentiable functions, Bull. Belg. Math. Soc. Simon Stevin 14 (2007), 425-444. Zbl 1165.26015 MR 2387040 
[5] R. W. Braun, R. Meise, and B. A. Taylor, Ultradifferentiable functions and Fourier analysis, Results Math. 17 (1990), 206-237. Zbl 0735.46022 MR 1052587

[6] T. Heinrich and R. Meise, A support theorem for quasianalytic functionals, Math. Nachr. 280 (2007), 364-387. Zbl 1130.46024 MR 2294806

[7] A. Kaneko, On the structure of Fourier hyperfunctions, Proc. Japan Acad. 48 (1972), 651653. Zbl 0262.46042 MR 0430264

[8] _ Introduction to hyperfunctions, Math. Appl. (Japan. Ser.) 3, Kluwer, Dordrecht, 1988. Zbl 0687.46027 MR 1026013

[9] A topological characterization of hyperfunctions with real analytic parameters, Sci. Papers College Arts Sci. Univ. Tokyo 38 (1988), 1-6. Zbl 0658.46034 MR 0954565

[10] K. Kataoka, On the theory of Radon transformations of hyperfunctions, J. Fac. Sci. Univ. Tokyo Sect. IA Math. 28 (1981), 331-413. Zbl 0576.32008 MR 0633002

[11] T. Kawai, On the theory of Fourier hyperfunctions and its applications to partial differential equations with constant coefficients, J. Fac. Sci. Univ. Tokyo Sect. I A Math. 17 (1970), 467-517. Zbl 0212.46101 MR 0298200

[12] O. Liess and Y. Okada, Support properties for integral operators in hyperfunctions, Adv. Math. 231 (2012), 1439-1461. Zbl 1271.46035 MR 2964611

[13] _ Ultra-differentiable classes and intersection theorems, Math. Nachr. 287 (2014), 638-665. Zbl 1302.46016 MR 3193941

[14] O. Liess, Y. Okada, and N. Tose, Hartogs' phenomena for microfunctions with holomorphic parameters, Publ. RIMS Kyoto Univ. 37 (2001), 221-238. Z Zbl 0993.32007 MR 1814566

[15] O. Liess and L. Rodino, Inhomogeneous Gevrey classes and related pseudodifferential operators, Boll. Un. Mat. Ital. C (6) 3 (1984), 233-323. Zbl 0557.35131 MR 0749292

[16] M. Sato, T. Kawai, and M. Kashiwara, Microfunctions and pseudo-differential equations, in Hyperfunctions and pseudo-differential equations (Katata, 1971), Lecture Notes in Math. 287, Springer, Berlin, 1973, 265-529. Z Zbl 0277.46039 MR 0420735 